www.jmscr.igmpublication.org

Index Copernicus Value: 79.54

ISSN (e)-2347-176x ISSN (p) 2455-0450

crossrefDOI: https://dx.doi.org/10.18535/jmscr/v7i2.163

\title{
Hyperfractionated Radio Therapy and Concurrent Chemotherapy with Cisplatin - Evaluation of immediate Response in locally Advanced Cancer Cervix
}

\author{
Author \\ B.Antoinette Mary Nithiya. M.D., RT \\ Email: nithiya1973@gmail.com
}

\begin{abstract}
Aim: Cancer cervix is the common malignancy among women globally. The Female population in developing countries is affected inspite of the Screening methods. The patients present in Locally advanced Stage due to Ignorance and Lack of awareness in wide geographic incidence spanning various continents. The aim of the Study is to assess the response with an aggressive treatment with Hyperfractionated Radio therapy and Concurrent cisplatin based chemotherapy in locally advanced Cancer cervix patients.

Patients and Methods: Most Patients belong to the Poor Socio Economic Stratum. Age group 30-60 Years with stage II B and Stage III B. Hyperfractionated Radio therapy 1.2 Gy bid (at 6 hours Interval) for 24 days upto 57.6Gy for 5days a week was given along with Concurrent Chemotherapy with cisplatin and was followed by HDR Brachytherapy 8 Gy in 2\#- 1 week apart. The response was assessed after 6 weeks of Completion of treatment

Results: Total no of cases studied were -24 II B cases -14 and IIIB cases - 10. The Overall complete response $-92 \%$ and Partial response - $8 \%$.

Conclusion: Invasive Cervical cancer is a Common cause of death in India. In view of the maximal therapy minimal time conceptual model concurrent Chemoradition appears to be ideal treatment to achieve maximal benefit the regimen uses gives better response in advanced stage, younger age group and lower Hemoglobin states at Presentation.

Keywords: Hyperfractionated Radiotherapy, Concurrent chemotherapy, brachytherapy, cancer cervix, cistplatin
\end{abstract}

\section{Introduction}

The major prerequisite for the development of cancer cervix are female sexual behavior and sexual intercourse. Women who start their sexual life at an early age particularly before 18 years are at 1.4 to 1.9 times increased risk of developing cancer cervix. Cancer cervix patients usually give a history of multiple sexual partners. The risk is doubled for women with 6 sexual partners. Risk factors related to parity include first childbirth at an early age and multiparity, the presence of which is associated with a higher incidence of cancer cervix in their spouses are sexual promiscuity: >3 extra marital partners, history of sexually transmitted disease, history of cancer penis or history of cancer cervix in first wife.

Among various agents, the HPV (Human Papilloma Virus) virus is considered to be the 
most likely candidate for etiological responsibility. Women who are HIV (Human immunodeficiency virus) positive have a 10 fold risk of cervical cancer in comparison with matched controls. Prevalence of cancer cervix in HIV positive patients below the age of 50 years is 19\%. There is much data suggesting an association between cancer cervix and HSV (Herpes simplex virus) but no conclusive proof is available.

\section{Previous Studies}

Clinicians have been investigating the use of concurrent chemoradiation for many years. The Gynecological Oncology Group has been investigating the role of concurrent chemoradiation in cancer cervix since the early 70's. The early studies concentrated on hydroxyurea a drug used as radiosensitiser. The effectiveness of Cisplatin in producing tumor regression in patients with local recurrence or distant metastasis after primary treatment paved the way for its use in combination with radiation at an earlier stage of treatment. From 1979 patients with advanced cancer cervix started receiving treatment with concurrent Cisplatin based chemoradiation. The overall 5 year survival of the study conducted by Blake et al was $49 \%$ for all stages.

A complete remission of $89 \%$ was observed for stage III disease. This study suggested that cisplatin based chemotherapy combined with radio therapy could be safely used to treat cancer cervix patients at high risk relax. Various prospective trials for patients with locally advanced cancer were under taken. Potish et al and Twiggs et al administered cisplatin weekly in an effort to increase the response. Fields et al and Runowicz et al conducted phase II trials of concurrent chemoradiation using Cisplatin (Dose $20 \mathrm{mg} / \mathrm{m} 2$ day $1-5$ at 21 day intervals). The data revealed better disease free survival and overall survival for Cisplatin based regimen. Souhami et al conducted a phase II Prospective trial of 50 patients with locally advanced cancer cervix patients stage IIA- IVA treated with concurrent chemoradiation $30 \mathrm{mg} / \mathrm{m} 2$ weekly one day of every week. The total dose to point A was 76Gy. A complete response rate $88 \%$ was seen. The actuarial survival rate at 4 years was $65 \%$ with acceptable toxicity. The studies like GOG 857,GOG -1208, GOG - 1239 RTOG -900110, SWOG -87 - 9711 showed a consistence advantage in complete response, DFS with reduction in mortality by $30-50 \%$ over conventional radiotherapy alone.

Hyperfractionated external radiotherapy with brachytherapy in bulky carcinoma cervix was studied in various trials by RTOG. RTOG -850512 trial shows that 1.2 Gy twice daily in ten fractions per week external radiotherapy administered to the whole pelvis with $4-5 \mathrm{hrs}$. interval between fractions up to a dose of $48 \mathrm{~Gy}$ followed by 1 or 2 intracavity applications to deliver a total minimum dose of $85 \mathrm{~Gy}$ at point $\mathrm{A}$ and $65 \mathrm{~Gy}$ to parametrium was equally effective as conventional RT was well tolerated and additional $10 \%$ parametrial dose was delivered. Since chemotherapy showed increased benefits and Hyperfractionated RT delivered more doses to the parametrium respecting the normal tissue toxicity attempts at combining chemotherapy with Hyperfractionated Radiotherapy were made. Calkins et.al assessed the toxicities of multiple daily fractionated whole pelvis radiation plus concurrent chemotherapy for locally advanced carcinoma of the cervix. Cisplatin $50 \mathrm{mg} / \mathrm{m} 2$ was administered on days 1 and 17 of external radiation, $5-\mathrm{FU}$ was given by the continuous IV infusion $(1 \mathrm{gm} / \mathrm{m} 2)$ for 4 consecutive days on 2 , $3,4,5$ and 18, 19, 20 and 21.The maximum tolerated dose of whole pelvis radiation that could be delivered in a Hyperfractionated setting with concomitant chemotherapy was 57.6Gy in 48 fractions followed by brachytherapy. RTOG -92-1014, this study was designed to administer twice daily radiation doses of $1.2 \mathrm{~Gy}$ to the pelvis and para- aortic at 4-6hr intervals five days per week. The total external radiation doses where $24-48$ Gy to the whole pelvis $12-36 \mathrm{~Gy}$ parametrical boost and $48 \mathrm{~Gy}$ to the para-aortic 
with an additional boost to a total dose of $54-58$ Gy to the known metastatic para -aortic site. 1 or 2 ICA where performed to deliver dose of $85 \mathrm{~Gy}$ to point A. Cisplatin (75 mg / m2, day 1 and 22) and $5 \mathrm{FU}$ ( 1000mg / m2 / 24hr x 4 days 1 and 22) were given for 2 or 3 cycles. The overall survival estimates were $59 \%$ at 1year and $47 \%$ at 2years. The study concluded unacceptably high rate $(31 \%)$ of grade four non hematological toxicity. The survival estimates appear no better than standard fractionation RT without chemotherapy. The National Cancer Institute issued a clinical announcement in 1999 stating that cisplatin based concurrent chemoradiation was the new standard of care in a locally advanced cancer cervix.

\section{Materials Methods \\ Eligibility Criteria}

Twenty four patients with locally advanced cancer cervix who satisfied the following eligibility criteria were included in this study

Age: 30 - 60 years; Disease stage: II B - III B

Performance status: Karnofsky performance score $\sim 80$

Histology: squamous cell carcinoma only

Hematological parameters

TC: 4000 and above / cubic mm

PLT: 1 lakh and above / cubic mm

RBC: 3 million and above / cubic mm

Hemoglobin> 10 gm $\%$

HIV negative

No history of treatment for the same complaints

\section{Therapeutic Protocol}

Hyperfractionated EBRT- Concurrent Chemotherapy: Hyperfractionated radiotherapy, 57.6Gy of EBRT 120cGy per fraction, twice daily at 6 hours interval for 5 days a week with Cisplatin based concurrent chemotherapy weekly, followed by Brachytherapy EBRT Equipment: Co - 60 Phoenix for Teletherapy ICA Equipment : HDR Remote after loader Ir- 192

Procedure of chemotherapy administration

Patient is pre- hydrated with one liter of Ringer lactate solution, 24 hours prior to commencement of chemotherapy during every cycle. On the day of chemotherapy, before administering the drug the patient is hydrated with $500 \mathrm{ml}$ of ringer Lactate solution. This was followed by injection of $4 \mathrm{mg}$ of Ondansetron, $50 \mathrm{mg}$ of Inj. Ranitidine and Inj Dexamethasone 8mg given, Manito 30 minutes prior to onset of Cisplatin administration. This was followed by infusion of $40 \mathrm{mg} / \mathrm{m} 2$ of Cisplatin dissolved in 1 litre of normal saline infused in 2 hours. This was followed by post chemo hydration with 1 litre of Normal saline. Finally $20 \mathrm{mg}$ of Inj. Frusemide was given i.v.

The entire procedure was completed in 4 hours. External beam radiation was delivered within 1 hour of chemotherapy then second fraction 6 hours later. Overall treatment time per patient is 52 days. The patients were to be reviewed every one month for the first six months followed by every 2 months for the next 2 years followed by once every 3 months thereafter.

\section{EBRT PROTOCOL}

Total dose delivered 57.6 Gy

Dose /\# $1.2 \mathrm{~Gy} /$ \#, 2\# a day 6 hours interval

No of fractions 48

Total duration 4 weeks and 4 days

Treatment days /week 5

Patients were assessed for ICA at the end of 48 fractions of EBRT.

\section{Bed Value Calculations for EBRT}

According to the linear-quadratic model the formula for calculating the biologically effective dose is

$$
\mathrm{BED}=\mathrm{Nxd}[1+\mathrm{d} /(\alpha / \beta)]-\mathrm{K}\left[\mathrm{T}-\mathrm{T}_{0}\right]
$$

where

$\mathrm{N}$ - no of fractions

d - dose per fraction

$\alpha / \beta$ - dose at which the linear and quadratic cell kills are equal

$\mathrm{k}$ - constant (Dose required /day to counter act proliferation)

$\mathrm{T}$ - Over all treatment time

$\mathrm{T}_{0}$ - Onset time for proliferation

In the conventional radiotherapy

Tumor - Cancerous tissue in the cervix 
$\mathrm{BED}=25 \times 2[1+2 / 10]-0.5[33-28]=57.5 \mathrm{~Gy}$

$(\alpha / \beta$ value of tumor in cervix-10 \& $\mathrm{k}$ is constant $0.5)$

Bladder- the late responding normal tissue $\mathrm{BED}=25 \times 2[1+2 / 4]=75(\alpha / \beta$ value of bladder4)

Rectum - the late responding normal tissue

$\mathrm{BED}=25 \mathrm{x} 2[1+2 / 3.87]=75.8 \quad(\alpha / \beta$ value of rectum-3.87)

In hyperfractionated radiotherapy

Tumor-cancerous tissue in the cervix

$\mathrm{BED}=48 \times 1.2[1+1.2 / 10]-0.5[32-28]=62.5 \mathrm{~Gy}$

$(\alpha / \beta$ value of tumor in cervix-10 \& $\mathrm{k}$ is constant -

$0.5)$

Bladder- the late responding tissue

$\mathrm{BED}=48 \times 1.2[1+1.2 / 4]=74.88(\alpha / \beta$ value of bladder -4)

Rectum - the late responding tissue

$\mathrm{BED}=48 \times 1.2[1+1.2 / 3.84]=75.5(\alpha / \beta$ value of rectum-3.87)

From the above calculations we find that by hyperfractionation we are able to escalate the dose to the tumor respecting the tolerance of the late responding normal tissues.

\section{HDR Brachytherapy Protocol}

Technique Remote after loading with Iridium-192 No of \# TWO (1 week after EBRT -1 week apart) Dose delivered to Point A 800cGy /\# -2\# (26Gy LDR equivalent)

\section{Summated Dose: EBRT \& HDR ICCA in the} Study

\begin{tabular}{|l|c|c|}
\hline & Location & Dose \\
\hline 1 & PT-A & 83.2 \\
\hline 2 & PT-B & $65 \mathrm{~Gy}$ \\
\hline 3 & Bladder & $<80 \mathrm{~Gy}$ \\
\hline 4 & Rectum & $<70 \mathrm{~Gy}$ \\
\hline
\end{tabular}

\section{Results}

After completing the full course of chemotherapy and radiation, the patients were analyzed for tumor regression along with assessment for toxic reactions 6 weeks after completion of treatment.

The response was based on clinical assessment, trans vaginal ultra sonogram, CT scan in patients. The response was graded as follows:
1) Complete Response No clinically detectable lesion.

2) Partial Response $50 \%$ regression of measurable tumor. No tumor area shows any progression No new area of lesion made out.

3) Static Response: No change in tumor size. No tumor area shows any progression. No new area of lesion made out

4) Progression: Increase in tumor size with treatment by $>25 \%$ or Appearance of lesions or Tumor induced death

Local control was defined as achievement of disease free period of at least 3 months after obtaining a complete response.

\section{Analysis of Treatment Results}

Analysis of immediate response was done 6 weeks after the end of treatment. This was done by clinical and radiological examination (pelvic and trans-vaginal USG and CT scan in some patients). All the 24 patients completed the prescribed treatments and were available for final evaluation of immediate response. The overall response of $100 \%$ seen at the end of external beam radiation was maintained after completion of treatment. None of the patients had static or progressive disease and there were no treatment-related deaths.

\section{Results of Study}

Totally 22 patients in the study attained complete response (92\% of the total patients in the arm). These included 13 out of 14 patients with stage IIB (93\% of all stage II B patients) and 9 out of the 10 patients with stage III B $(90 \%$ of all stage III B patients).The study benefited patients with KPS of $>90 \%$ with a CR of $100 \%$ followed by patients with a KPS of 80 with a CR of $86 \%$.The complete response rate increased with increasing levels of hemoglobin. The trial benefited patients with $\mathrm{Hb}$ levels of $>11 \mathrm{gm} \mathrm{\%}$ with a CR of $100 \%$ in this group.

The complete response was attained in well differentiated as well as in poorly differentiated types with not much of significance.

Patients with unilateral disease had $100 \%$ complete response while those with bilateral 
disease had a complete response of $87 \%$. Thus patients with unilateral disease are found to have better response rates. The present study shows that escalating the dose to the tumor by using hyper fractionation results in better response rates in higher stages of the tumor.

Patients with stage IIB and unilateral parametrial disease had $100 \%$ CR.

Patients with stage IIB and bilateral parametrial disease had $80 \% \mathrm{CR}$.

Patients with stage III B and bilateral parametrial disease had $90 \% \mathrm{CR}$

Thus patients with bilateral parametrial involvement and stage III B disease benefited from study protocol.

\section{Analysis of Partial Responders}

Analysis revealed that the factors associated with a higher rate of partial response (PR) were:

1. AGE $-<50$ years

2. PERFORMANCE - KPS of $<90 \%$

3. $\mathrm{Hb}$ STATUS $-\mathrm{Hb}$ value of $<11 \mathrm{gm} \%$

4. STAGE - III B

5. PARAMETRIAL DISEASE - Bilateral parametrial disease

\section{Results of Study}

\begin{tabular}{|l|c|c|c|c|c|}
\hline \multirow{2}{*}{ Stage } & \multirow{2}{*}{ No of pts } & \multicolumn{2}{|c|}{ CR } & \multicolumn{2}{c|}{ PR } \\
\cline { 3 - 6 } & & No & \% & No & \% \\
\hline II B & 14 & 13 & 93 & 1 & 7 \\
\hline III B & 10 & 9 & 90 & 1 & 10 \\
\hline Total & 24 & 22 & 92 & 2 & 8 \\
\hline
\end{tabular}

Response vs. Age of Patient

\begin{tabular}{|l|c|c|}
\hline \multirow{2}{*}{ Age } & \multicolumn{2}{|c|}{ No. of patients(\%) } \\
\cline { 2 - 3 } & CR & PR \\
\hline $31-40$ & $8(89 \%)$ & $1(11 \%)$ \\
\hline $41-50$ & $8(89 \%)$ & $1(11 \%)$ \\
\hline $51-60$ & $6(100 \%)$ & - \\
\hline
\end{tabular}

\section{Response vs. Performance Status (KPS)}

\begin{tabular}{|l|c|c|}
\hline \multirow{2}{*}{ KPS } & \multicolumn{2}{|c|}{ No. of patients(\%) } \\
\cline { 2 - 3 } & CR & PR \\
\hline 90 & $10(100 \%)$ & - \\
\hline 80 & $12(86 \%)$ & $2(14 \%)$ \\
\hline
\end{tabular}

\section{Response Vs HbStatus}

\begin{tabular}{|l|l|l|}
\hline \multirow{2}{*}{ Hb } & \multicolumn{2}{|l|}{ No. of patients(\%) } \\
\cline { 2 - 3 } & CR & PR \\
\hline $10-11 \mathrm{~g} / \mathrm{dl}$ & $18(90 \%)$ & $2(10 \%)$ \\
\hline $11.1-12 \mathrm{~g} / \mathrm{dl}$ & $4(100 \%)$ & - \\
\hline
\end{tabular}

\section{Response Vs HPE}

\begin{tabular}{|l|c|c|}
\hline \multirow{2}{*}{ HPE } & \multicolumn{2}{|c|}{ No. of patients(\%) } \\
\cline { 2 - 3 } & CR & PR \\
\hline Well differentiated & $2(100 \%)$ & $0(0 \%)$ \\
\hline Moderately differentiated & $17(89 \%)$ & $2(11 \%)$ \\
\hline Poorly differentiated & $3(100 \%)$ & $0(0 \%)$ \\
\hline
\end{tabular}

\section{Response vs Parametrial Involvement}

\begin{tabular}{|l|c|c|}
\hline \multirow{2}{*}{ P.M. involvement } & \multicolumn{2}{|c|}{ No. of patients(\%) } \\
\cline { 2 - 3 } & CR & PR \\
\hline Unilateral & $9(100 \%)$ & - \\
\hline Bilateral & $13(87 \%)$ & $2(13 \%)$ \\
\hline
\end{tabular}

\section{Response vs. Disease Stage}

\begin{tabular}{|l|c|c|}
\hline \multirow{2}{*}{ Stage } & \multicolumn{2}{|c|}{ No. of patients(\%) } \\
\cline { 2 - 3 } & CR & PR \\
\hline II B & $13(93 \%)$ & $1(7 \%)$ \\
\hline III B & $9(90 \%)$ & $1(10 \%)$ \\
\hline
\end{tabular}

\section{Discussion}

Invasive cervical cancer is the commonest cause of death from cancer among women in India. The constraints of the single modality regimens in achieving a "cure" forces to venture into newer modalities of treatment to achieve this goal. In view of the "maximal therapy - minimal time" conceptual model, concurrent chemoradiation appears to be ideal treatment to achieve maximal benefit within the "window of curability". Several randomized clinical trials stand to prove the same. The NCI 'Clinical announcement' in Feb 1999 suggesting that 'strong consideration should be given to the incorporation of concurrent Cisplatin based chemotherapy with radiation therapy in treatment of locally advanced cancer cervix reiterates the same.

Cisplatin has been the chemotherapeutic agent of choice both as a single agent and in combination with other drugs in concurrent chemoradiation. The synergism between Cisplatin and radiotherapy has been well established.

The reported response rates of concurrent chemoradiation with various Cisplatin based regimes vary from $74 \%-100 \%$ with complete response rates of $63 \%-88 \%$. Cisplatin at the present time is considered to be the single most cytotoxic agent in the treatment of locally advanced cancer cervix. We did a detailed analysis of the patient and 
tumor characteristics during the evaluation of results. We found a better understanding of these factors will lead to more rational therapeutic strategies with better optimization of radiation doses alone or various combinations of radiation with other modalities of management which will yield the highest pelvic tumor control with acceptable toxicity thus tailoring to the patient needs. This approach has been advocated by Fletcher and others and involves the definition of appropriate doses of radiation for a given tumor volume (and not based on clinical stage), in specific patients (according to age and KPS), choice of treatment modality (irradiation alone or concurrent chemoradiation) based on tumor bulk and parametrial extension.

\section{Analysis of Radiation Protocol}

The RTOG protocol with hyper fractionation increases the parametrial dose by $10 \%$.As all our patients had locally advanced disease we initially treated all our patients with a dose of $57.6 \mathrm{~Gy}$ delivered to the whole pelvis. We proceeded with a dose to point A 83.2Gy keeping in mind factors such as the normal tissue tolerance and the higher probability of acute and late toxicity with combined modality treatment. As most of the patients in trial arm had bilateral parametrial disease we proceeded with a higher dose of $65 \mathrm{~Gy}$ to the parametrium. We saw that the overall treatment in both arms irrespective of brachytherapy modality was completed within 8 weeks.

The Biologically Effective Dose (BED) value of 82.3 Gy to point A used in this protocol is $88 \mathrm{~Gy}$ 10.

\section{Analysis of Chemotherapy Protocol}

We used the weekly CDDP regimen which is commonly used in the concurrent setting. The aim of this chemotherapy schedule was to observe whether the relatively low dose of weekly CDDP used produced the same immediate response rates and toxicity in the Indian population as had been reported in the western literature. RT dose and chemo schedule were modified in such that they produced acceptable acute toxicity with scope for dose escalation. We also did not have any patient dropping out of the study due to toxic effects.

\section{Discussion of Treatment Results}

The detailed analysis of the case results has enabled us to identify certain tumor / patient characteristics which alter the immediate therapeutic response. The method of statistical analysis adopted for the present study is the Chi square test. This test is used to find the association between two variables. The Chi- square distribution is determined by a single parameter, the degrees of freedom which are equal to (r-1) (c1).

\section{Conclusion}

Stage of disease, volume of disease, general condition of the patient, performance status are well recognized prognostic factors which influence therapeutic outcome in cancer of the cervix. The sub-group of patients with added benefits over the concurrent chemoradiation studies with acceptable toxicities are i. Stage IIIB disease ii. Younger patient's iii Lower hemoglobin status. The only toxicity observed was $87 \%$ grade-1 leucopenia. The fall in WBC counts was transient and the patients recovered without any treatment. It was observed that the bone marrow suppression was more in the study due to the combined effect of increased RT dose to the pelvic hematopoietic tissues and chemotherapy. The patients are on regular follow up for accrual of long term results. The impact of this regime on long term survival as well as the long term morbidity associated with this protocol is to be analyzed in the future.

\section{Source of fund: nil}

Conflict of interest: none declared

\section{References}

1. Blake et al - Combined radiotherapy and chemotherapy for advanced carcinoma of the cervix. Clinical radiology 37(5):465- 469 Sep 1986. 
2. Potish et al - Effect of Cisplatin on tolerance to radiation therapy in advanced cervical cancer. American journal of clinical radiology. Vol 9:387-391, 1986.

3. Twiggs et al - Concurrent weekly Cisplatin and radiotherapy in advanced cervical Cancer. Gynecol oncology 24: 143-148, 1986. 61:416-422,

4. Fields et al - Mature results of a phase- II study on concomitant Cisplatin\& pelvic radiotherapy in locally advanced cervical cancer. Gynec oncology 1996.

5. Runowicz et al - Concurrent Cisplatin and radiotherapy in locally advanced cancer cervix. Gynec oncology -34:395-401, 1989

6. Souhami et al - Weekly Cisplatin and external beam radiation and HDR brachytherapy in locally advanced cancer cervix. Int J RadiatOncolBiol Phys.

7. Whitney et al-GOG 85 report. Journal of clinical oncology .Vol- 17:13391345, 1999.

8. Peter.G. Rose et al - GOG 120 report. NEJM 340(15)1144-1153, April 1999.

9. Henry .M. Keys et a1 - GOG 123 report. NEJM 340( 15) 1154- 1161, April 1999.

10. Mitchell. Morris et a1 RTOG- 90-01 report. NEJM 340(15)1137- 1143, April 1999.

11. Peters et a1 SWOG-87-97 report- Gynec oncology 72: 443-450, 1999.

12. Ritsukokomaki M.D, Thomas F.Pajak, Victor A Marcial et. al Twice daily fractionation of external irradiation with brachytherapy in Bulky carcinoma of cervix RTOG 88-05 Cancer 1994 : 73 : 2619- 25.

13. Alison R Calkins.M.D et.al Hyperfractionated Radiation Therapy plus chemotherapy in locally advanced cervical cancer Gynecologic oncology 5:3:349-355 1999.

14. Perry. W. Gregsby .J.D.LU: David Mutch ; Robert Y Kim patricia J Eifel.et.al Twice daily fractionation of external irradiation with brachytherapy and chemotherapy in carcinoma of cervix with positive para - aortic lymph nodes Gynecology cancer working group- Int Jou. Rad Oncology, Biology and physics51(3) 58-59, 2001.

15. R.Baskaran et.al Toxicities and tolerance of the Cancer Cervix patients Asian journal of Pharmaceutical and Clinical Research.Vol.4 Issue 1, 2011. 